\title{
Culture Into Perfusion-Assisted Bioreactor Promotes Valve-Like Tissue Maturation of Recellularized Pericardial Membrane
}

\author{
Francesco Amadeo ${ }^{1 t}$, Marianna Barbuto ${ }^{1 t}$, Giacomo Bernava ${ }^{1 \dagger}$, Nicla Savini ${ }^{1}$, \\ Maura Brioschi ${ }^{2}$, Stefano Rizzi ${ }^{1}$, Cristina Banfi ${ }^{2}$, Gianluca Polvani ${ }^{3}$ and Maurizio Pesce ${ }^{\text {1*}}$ \\ ${ }^{1}$ Unità di Ingegneria Tissutale Cardiovascolare, Centro Cardiologico Monzino, IRCCS, Milan, Italy, ${ }^{2}$ Unità di Proteomica, \\ Centro Cardiologico Monzino, IRCCS, Milan, Italy, ${ }^{3}$ Dipartimento di Scienze Cliniche e di Comunità, Università degli studi di \\ Milano, Milan, Italy
}

OPEN ACCESS

Edited by:

Gianfranco Pintus,

University of Sharjah, United

Arab Emirates

Reviewed by:

Sharan Ramaswamy,

Florida International University,

United States

Lucio Barile,

University of Zurich, Switzerland

*Correspondence:

Maurizio Pesce

maurizio.pesce@ccfm.it

tThese authors have contributed equally to this work

Specialty section

This article was submitted to

Cardiovascular Biologics and Regenerative Medicine,

a section of the journal

Frontiers in Cardiovascular Medicine

Received: 10 February 2020 Accepted: 15 April 2020

Published: 12 May 2020

Citation:

Amadeo F, Barbuto M, Bernava G, Savini N, Brioschi M, Rizzi S, Banfi C, Polvani $G$ and Pesce M (2020) Culture Into Perfusion-Assisted Bioreactor Promotes Valve-Like Tissue Maturation of Recellularized

Pericardial Membrane.

Front. Cardiovasc. Med. 7:80. doi: 10.3389/fcvm.2020.00080
Derivation of tissue-engineered valve replacements is a strategy to overcome the limitations of the current valve prostheses, mechanical, or biological. In an effort to set living pericardial material for aortic valve reconstruction, we have previously assessed the efficiency of a recellularization strategy based on a perfusion system enabling mass transport and homogenous distribution of aortic valve-derived "interstitial" cells inside decellularized pericardial material. In the present report, we show that alternate perfusion promoted a rapid growth of valve cells inside the pericardial material and the activity of a proliferation-supporting pathway, likely controlled by the YAP transcription factor, a crucial component of the Hippo-dependent signaling cascade, especially between 3 and 14 days of culture. Quantitative mass spectrometry analysis of protein content in the tissue constructs showed deposition of valve proteins in the decellularized pericardium with a high variability at day 14 and a reproducible tissue maturation at 21 days. These results represent a step forward in the definition of strategies to produce a fully engineered tissue for replacing the calcified leaflets of failing aortic valves.

Keywords: biomaterials, valve implant, pericardium, valve interstitial cell, perfusion system

\section{INTRODUCTION}

Diseased and dysfunctional heart valves are routinely replaced by surgical intervention. About 300,000 heart valve procedures are performed annually worldwide; this number is expected to triple by 2050 with the majority of the patients over the age of $65(1,2)$. Despite their nonmodifiable mechanical performance, commercially available mechanical prostheses are prone to thromboembolic complications causing patients to require lifelong anti-coagulation therapy. Biological valves, produced with animal-derived pericardium and valves, or deriving from tissue homografts, undergo structural deterioration, and this is still the principal cause of their failure in the mid/long term. This problem affects significant proportions of patients, especially the young, which mandates re-intervention (3). Deterioration of the biological implants is caused primarily by chronic inflammatory reaction due to the failure to detoxify the fixative remnants in the tissue $(4,5)$, and/or the incomplete removal of major xeno-antigens (6-10) ( $\alpha$-Gal). A current solution to circumvent these problems consists of the design of tissue engineered heart valves (TEHVs) by combining biodegradable scaffolds and cells of various origins $(11,12)$. Despite several materials 
and cell types have been proposed, there are still a number of unresolved problems in TEHVs due to insufficient structural stability of the engineered leaflets and consequent leaflet "retraction" and "thickening" effects (13), which result in TEHVs failure at mid-/long-term (14).

In previous contributions $(15,16)$, we showed the suitability of a decellularization procedure with ionic/non-ionic detergents to maintain the mechanical properties and reduce the immunogenicity of porcine pericardium. Besides drastically reducing the content of xenoantigens, the treatment also increased the permeability of the tissue, thus making possible the employment of a perfusion bioreactor to enable mass transport through the pericardial matrix and promote stable cellularization (16). Compared to other recellularization techniques to seed cells in valve-competent scaffolds based on static culture $(17,18)$, the employment of this system enabled a higher penetration of valve interstitial cells (VICs) inside the scaffold and reduced the activation of these cells into myofibroblasts. The aim of the present investigation was to assess the maturation of the "living" pericardium material, particularly concerning the protein deposition inside the matrix and the cellular phenotype at short and long culture time points, in view of scaling up the procedure for clinical transfer.

\section{METHODS \\ Cell Culture}

Primary aortic VICs were isolated from porcine aortic valve as previously described (15). Cells were cultured up to four passage in Dulbecco's modified Ealge's medium (DMEM, Lonza) containing 1\% L-Glutamine (Lonza), 1\% penicillin-streptomycin (Lonza), and 10\% fetal bovine serum (FBS, Thermo Scientific).

\section{Decellularization of Porcine Pericardium}

Pericardium samples were obtained from four porcine hearts explanted at an authorized slaughterhouse. Decellularization of the tissue was performed according to the optimized protocol described in a previous work (16). Briefly, the pericardium was cut from corresponding left ventricular portion and the adipose tissue was mechanically removed from the surface of the heart. After washing with a sterile solution of phosphate buffered saline (PBS) without $\mathrm{Ca}^{2+}-\mathrm{Mg}^{2+}$ containing Aprotinin (2 $\mu \mathrm{l} / \mathrm{mL}$, Trasylol, Bayer) at $4^{\circ} \mathrm{C}$ in agitation for $90 \mathrm{~min}$, the tissue was incubated in a hypotonic buffer solution with $10 \mathrm{mM}$ Tris- $\mathrm{HCl}$ ( $\mathrm{pH} 8$ ), ethylenediamine tetraacetic acid (0.1\%, EDTA), and Aprotinin $(2 \mu \mathrm{l} / \mathrm{mL})$ at $4^{\circ} \mathrm{C}$ for $16 \mathrm{~h}$ under continuous agitation. The tissue was then washed with Milli-Q for $90 \mathrm{~min}$ (under agitation at room temperature, RT), and incubated in 1\% of Triton X-100 (Sigma) solution at RT for $24 \mathrm{~h}$, followed by extensive rinsing in Milli-Q water for $90 \mathrm{~min}$ (RT, in agitation), and incubation in $0.1 \%$ sodium dodecyl sulfate (SDS) solution at RT for $24 \mathrm{~h}$. The tissue was finally treated with DNAse $(50 \mathrm{U} / \mathrm{mL})$ into a PBS $\mathrm{Ca}^{2+}-\mathrm{Mg}^{2+}$ solution for $90 \mathrm{~min}$ at $37^{\circ} \mathrm{C}$. Before the culture tissue was sterilized by incubation in BASE.128 (a tissue factory approved sterilization medium) at $4{ }^{\circ} \mathrm{C}$ for $72 \mathrm{~h}$.

\section{Dynamic Seeding and Tissue Maturation Through Perfusion Bioreactor}

Following the same procedure described by Amadeo et al. (16), decellularized porcine pericardium patches $(1 \mathrm{~cm}$ diameter) were housed into bioreactor chambers and after that, the bioreactors were assembled. VICs $\left(6 \times 10^{5}\right.$ cells/bioreactor $)$ suspension $(10 \mathrm{~mL})$ were dynamically seeded for 3 days applying a high flow at $3 \mathrm{~mL} / \mathrm{min}$. After this phase, the recellularized patches were cultured up to 21 days under a low flow rate of $0.03 \mathrm{~mL} / \mathrm{min}$ (Figure 1A). During the whole process, the alternate flow was applied to enhance nutrient supply and gas exchange. The culture medium was partially $(6 \mathrm{~mL})$ changed every 3 days.

\section{Characterizations of Living Tissue}

For the biological characterization of the tissue, three time points were chosen, corresponding to 3,14, and 21 days of dynamic cell culture. To evaluate the vitality of the cells seeded into the decellularized pericardium, recellularized samples were incubated in $400 \mu \mathrm{L}$ of $1.2 \mathrm{mM} 3$-(4,5-dimethylthiazol-2-yl)2,5-diphenyltetrazolium bromide (MTT) solution at $37^{\circ} \mathrm{C}$ for $3 \mathrm{~h}$. After that time, images of both sides of the constructs were acquired through Stemi 2000-C Stereo Microscope (Carl Zeiss). For histological analyses, porcine aortic valves and recellularized samples were fixed in $4 \%$ paraformaldehyde $\left(4^{\circ} \mathrm{C}\right.$ overnight) before including in paraffin. Histological sections ( $5 \mu \mathrm{m}$ thickness) were cut, dewaxed and stained with Masson's trichrome staining (Bio Optica). Images were acquired using an AxioScope optical microscope (Carl Zeiss). Immufluorescence on recellularized pericardium was performed using antigen retrieval solution ( $10 \mathrm{mM}$ sodium citrate buffer, $0.05 \%$ Tween-20, $\mathrm{pH}$ 6) and permeabilization with Triton X-100 (1\% v/v) bovine serum albumin (BSA, 3\% w/v, Sigma) solution in PBS. Mouse and rabbit anti-Alpha-Smooth Muscle Actin ( $\alpha$ SMA), rabbit antivimentin, mouse anti-YES-Associated Protein (YAP), and mouse anti-Proliferating Cell Nuclear Antigen (PCNA) were chosen as primary antibodies to evaluate the phenotype of the VICs into the pericardium constructs. The incubation with primary antibodies ( $\alpha$ SMA; rabbit, $2 \mu \mathrm{g} / \mathrm{mL}$, Abcam - $\alpha \mathrm{SMA}$; mouse, $0.7 \mu \mathrm{g} / \mathrm{mL}$, DAKO - PCNA, mouse, $2 \mu \mathrm{g} / \mathrm{mL}$, DAKO - YAP, mouse, $2 \mu \mathrm{g} / \mathrm{mL}$, Santa Cruz Biotechnology, Vimentin, rabbit, $0.45 \mu \mathrm{g} / \mathrm{mL}$, Cell Signaling) was carried out in a PBS solution containing BSA $(3 \% \mathrm{w} / \mathrm{v})$ overnight at $4^{\circ} \mathrm{C}$. After thorough washing with abundant PBS, sections were incubated with 488-donkey anti-mouse $(10 \mu \mathrm{g} / \mathrm{mL}$, Invitrogen $)$ and 594-goat anti-rabbit $(10 \mu \mathrm{g} / \mathrm{mL}$, Invitrogen) secondary antibodies. Cell distribution in the constructs and cells counting were assessed by $4^{\prime}$, 6-diamidino-2-phenylindole (DAPI, $100 \mu \mathrm{g} / \mathrm{mL}$,Sigma) nuclei staining. All the images were acquired with Apotome fluorescence microscope (Carl Zeiss) or laser scanning confocal microscope (Carl Zeiss).

\section{Proteomic Assessment}

Recellularized samples were snap frozen immediately after unmounting from the culture bioreactors. For proteomic analysis, three independent patches for each time/pericardia samples were stored. Protein extraction was performed for all samples in the same run as follows: homogenization in an 
A

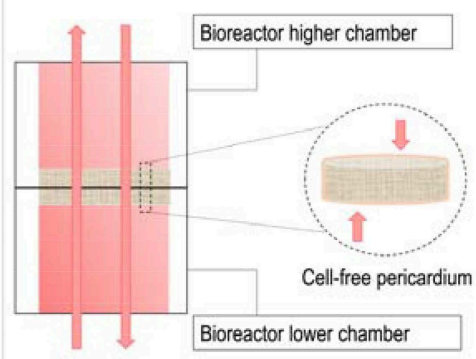

Alternate Medium flow

Dynamic cell seeding [ $3 \mathrm{ml} / \mathrm{min}]$

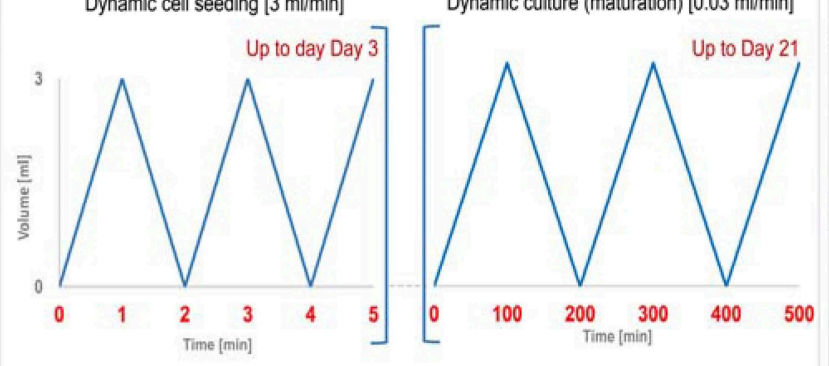

B

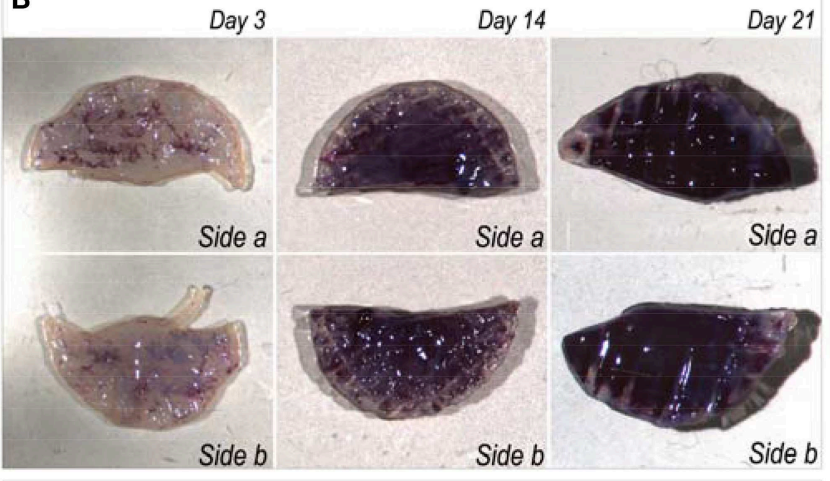

C

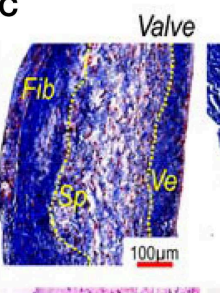

Valve

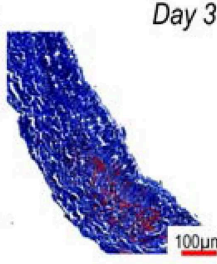

Day 3

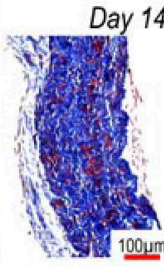

Day 14
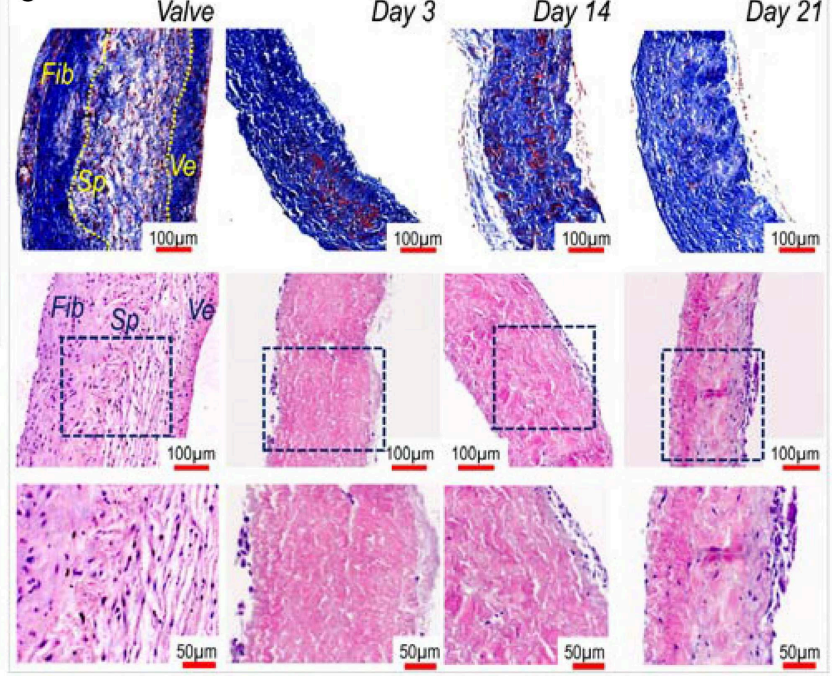

FIGURE 1 | Dynamics of pericardium recellularization process. (A) The upper part of the figure describes the alternate motion of the culture medium flow in the chamber of the perfusion bioreactor. The lower part indicates time-dependent representation of the flow during the cell seeding phase (left graph) occurring for the first 3 days and the maturation phase (right graph). (B) MTT staining of pericardial patches at different recellularization times shows effective growing of valve cells inside the pericardial matrix up to 21 days of culture. (C) Imaging of transversally sectioned recellularized pericardial samples at different times of culture stained with trichrome Masson's (top) and hematoxylin/eosin (bottom). As a comparison, sectioned native aortic valve tissue is included, where a distinction between the three layers, the Fibrosa (Fib), the Spongiosa (Sp), and the Ventricularis (Ve), is shown. Images in the bottom are magnifications of the insets highlighted by blue boxes in the middle panels.

extraction buffer containing Tris $0.1 \mathrm{M}$ pH 7.6 and SDS $4 \%(400$ $\mu \mathrm{L}$ of buffer for $50 \mathrm{mg}$ of tissue) performed with the UltraTurrax at $24,000 \mathrm{rpm}$ for $30 \mathrm{~s}$ on ice, sonication (10 s twice power 6 on ice), and heating at $95^{\circ} \mathrm{C}$ for $3 \mathrm{~min}$. For label-free quantitative mass spectrometry (LC-MSE), protein extracts were precipitated with the protein precipitation kit (Calbiochem), according to the manufacturer's instructions. Protein pellets were then dissolved in $25 \mathrm{mmol} \mathrm{NH}_{4} \mathrm{HCO}_{3}$ containing $0.1 \%$ RapiGest (Waters Corporation), and digested with trypsin as previously described (19). LC-MSE analysis was performed as previously described and analyzed with Progenesis QIP v4.1 (Non-linear dynamics) including normalization of protein abundance considering all the identified proteins and Principal Component Analysis (20, 21). UniProt database (release 2017-6; number of sequence entries for sus scrofa, 3,549) was used for database searches.

\section{Images and Statistical Analysis}

All the data are represented as mean \pm standard error. Differences among experimental groups were assessed by GraphPad statistical Software. The type of statistical tests and the number of the replicates included in the analysis are specified in the figure legends.

\section{RESULTS}

As previously shown by us (16), dynamic seeding with an alternate perfusion bioreactor is a promising method to recellularize aldehyde-free decellularized pericardium (see characteristics of the seeding/culture protocol in Figure 1A). In keeping with these findings, porcine VICs penetrated inside the inner part of the decellularized pericardium with a constant increment in MTT color staining on both sides of the patches 


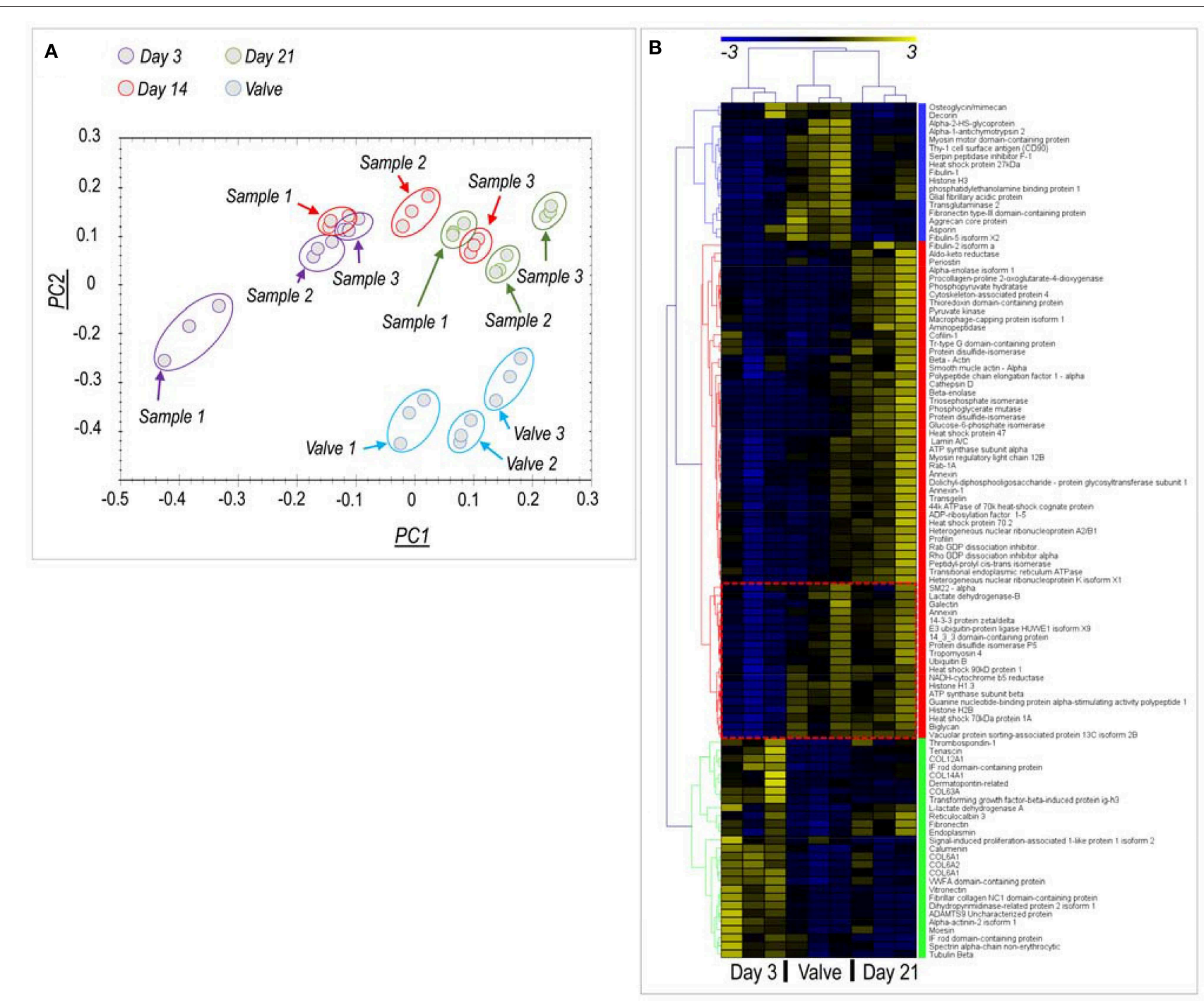

FIGURE 2 | Proteomic analysis of native valves and recellularized pericardial samples. (A) Principal component (PC) analysis of protein content in the analyzed samples. Three independent pericardia were recellularized with pig-derived VICs and analyzed at each time point (Samples \#1- \#3). Each of these samples is represented by experimental triplets evidenced by time-specific color code, for a total of nine samples per time point. Three porcine aortic valves tissues were analyzed in parallel (also in triplets) and are also indicated in the PC analysis. As shown, the alignment of the PC1 recellularized samples at day 21 with the PC1 of the valve tissue reveals a partial restoring of the native protein content by VICs. (B) Clusterization analysis of proteins differentially expressed at day 3 and day 21 vs. the native tissue (see Table $\mathbf{S} \mathbf{1}$ for description of the proteins and Figure $\mathbf{S} \mathbf{1}$ for the analysis including day 14 samples). Three protein clusters (each highlighted by a different color) were identified. A first cluster (characterized by the blue color) contains proteins that were expressed at higher levels in the native valves vs. the recellularized samples independently of time. A "red" cluster contained proteins whose expression was relatively higher at day 21 stage than in valve or day 3 samples. Interestingly, a sub-cluster of these proteins (encircled in the heatmap by a red box) were contained at equal levels in day 21 and valve tissues. A "green" cluster containing proteins overrepresented in day 3 recellularized samples was finally found, mainly constituted by structural matrix components. As discussed, this higher abundance might be explained by a lower protein content due to the presence of few cells, which cannot compensate for the protein loss due to decellularization procedure, given the short culture time.

(Figure 1B), and this preserved tissue structural integrity with possible de novo synthesis of extracellular matrix components (Figure 1C). We already showed that a mass spectrometry-based approach is useful to assess the composition of the pericardial matrix before and after decellularization (16). Therefore, here we employed the same technique to get insights on the maturation process of the extracellular matrix in consequence of cells seeding. MS analysis of native porcine valves and recellularized pericardium at different time points rendered a list of 105 proteins (Table S1) that were differentially expressed at different culture times and vs. the native valve tissue. As shown in the table, there were protein groups that were present at specific stages during the maturation of the cellularized tissue in the bioreactor, in addition to one group of proteins that were more abundant in the native valves vs. all the recellularized samples, irrespective of the maturation stage. 
A
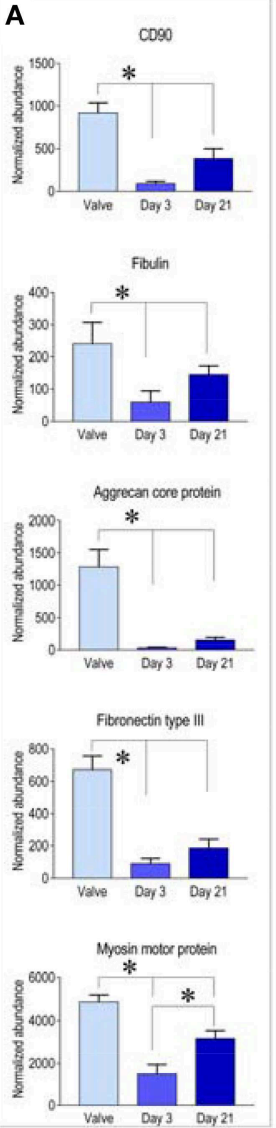
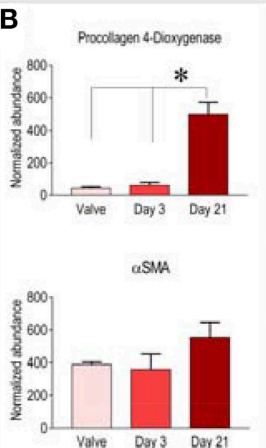

SMR2a
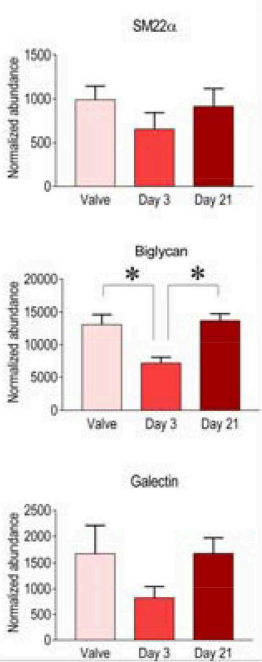
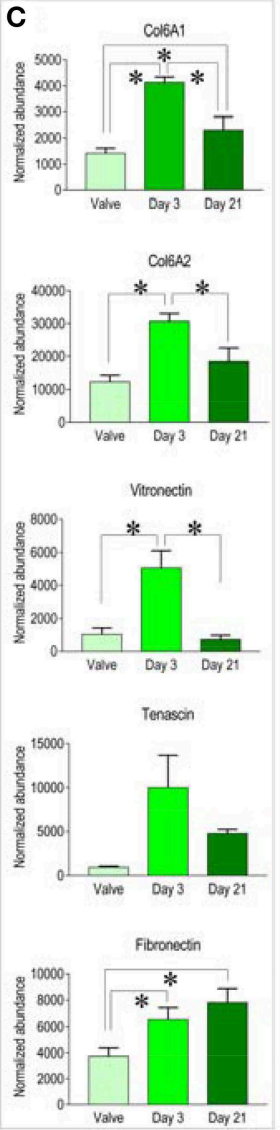

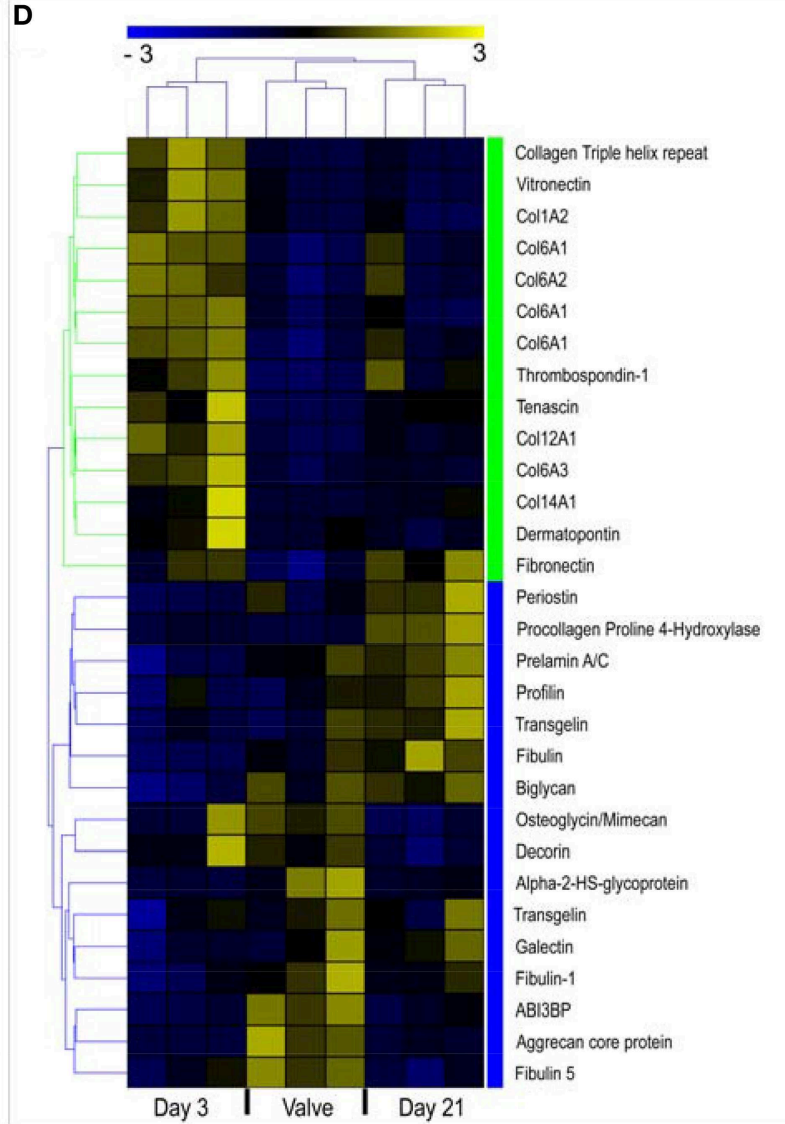

FIGURE 3 | (A-C) Example of graphic representation of selected proteins belonging to the three clusters identified by the analysis of proteomic data. Statistical analysis was performed by one way ANOVA with Newman-Keuls post-hoc on the normalized abundance of proteins identified by mass-spectrometry (see Table S1). * represents $P<0.05(n=3)$. (D) Clusterization analysis of proteins with functions in ECM structure/remodeling. Treatment of this data in a separate analysis helped to assess in better details the representation of matrix-related protein distribution during the course of the recellularization procedure. Although separate ECM protein clusters were still identified for the three tissue types, the heatmap clearly shows a higher separation between the day 3 samples and the day $21 /$ native valve tissues, and the higher similarity of the day 21 samples to native valves. This last evidence suggests a trend of the day 21 samples to evolve toward a valve-like tissue.

Principal component analysis and clusterization of normalized protein levels Figure 2A indicated a good reproducibility of the recellularization process in the three replicates analyzed for each independently recellularized pericardium samples; in addition, a good data separation was observed between day 3 and day 21 samples, while a partial overlapping of the protein groups representing the day 14 samples and those at the two other culture times was evident. Unsupervised data analysis also showed clusterization of proteins expressed in native valves, day 3 and day 21 samples, and a wider dispersion of data for the day 14 time point (Figure S1).

To analyze better the evolution of the protein content of pericardial samples between the beginning and the end of the culturing period, we eliminated from the analysis the day 14 time-point and performed another data clusterization run, taking into account only the day 3 , day 21 , and the native valve data. Under these conditions (Figure 2B), proteomic data clearly indicated protein clusters that discriminated each of the three experimental conditions. Interestingly, the number of proteins expressed at the final end point of the culture (day 21) (Red cluster in Figure 2B; $n=59$ proteins), was higher than the number of proteins more abundant in native valves (Blue cluster in Figure $\mathbf{2 B}, n=20$ proteins) or day 3 recellularization stage (Green cluster in Figure $\mathbf{2 B}, n=22$ proteins) together. In particular, it was noted the presence of a protein sub-cluster in the red group (encircled in Figure 2B) where the protein content was comparable in day 21 recellularized samples and native tissues. This cluster contained, among others, important cellular proteins such as Ubiquitin and Histone H1.3.

Analyzing data more in details, and directing our interest on relevant cellular proteins for the valve cells and tissues, we found higher expression of the mesenchymal stem cell marker CD90 (Thy1) and of Fibulin, Aggrecan, and Fibronectin III, three ECM components particularly abundant in the native valve samples compared to the pericardial samples at both culture times. On the other hand, a similar analysis performed on representative proteins of the red cluster (Figure 2B) indicated presence of proteins involved in ECM 


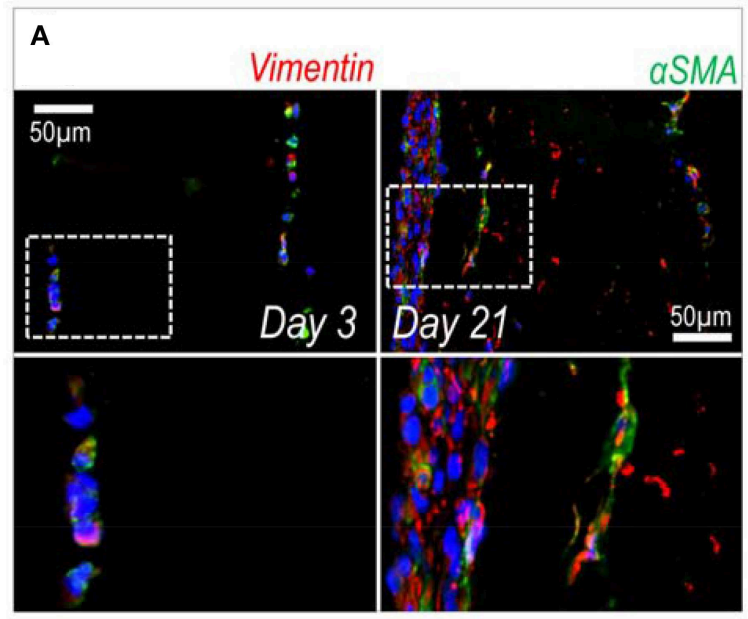

D

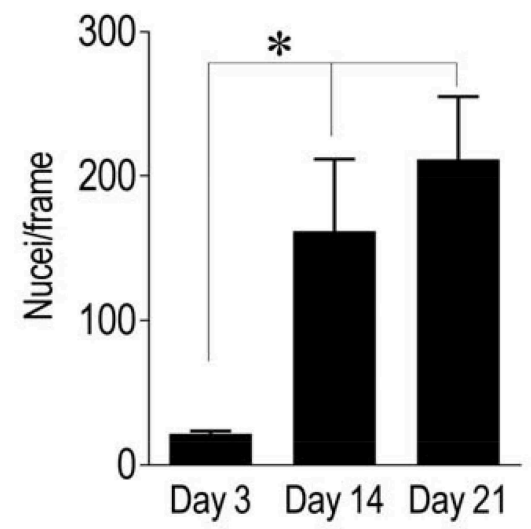

B

Vimentin

PCNA
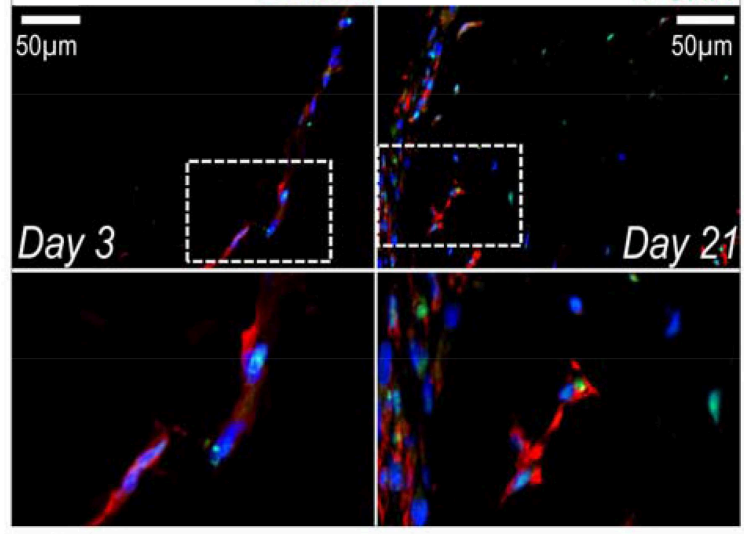

C
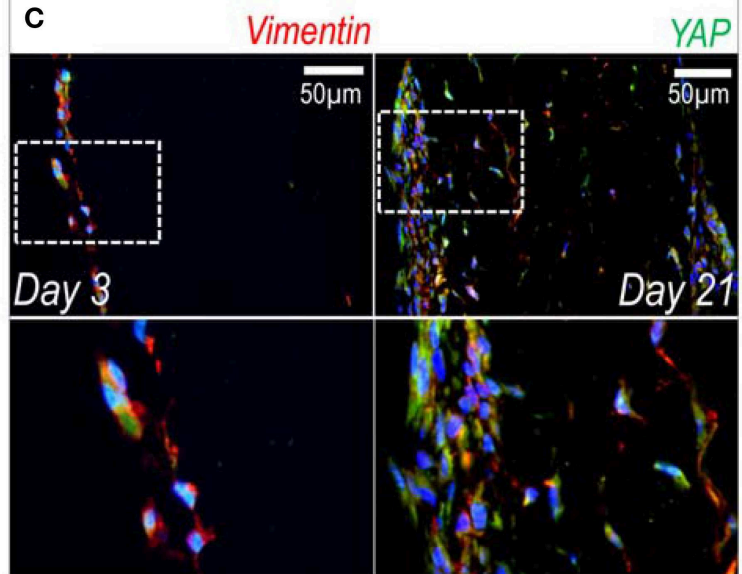

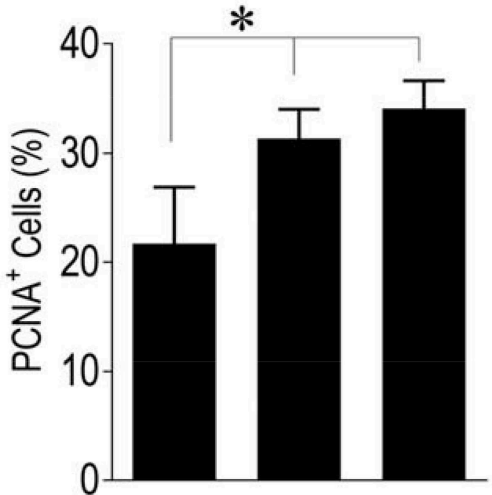

Day 3 Day 14 Day 21

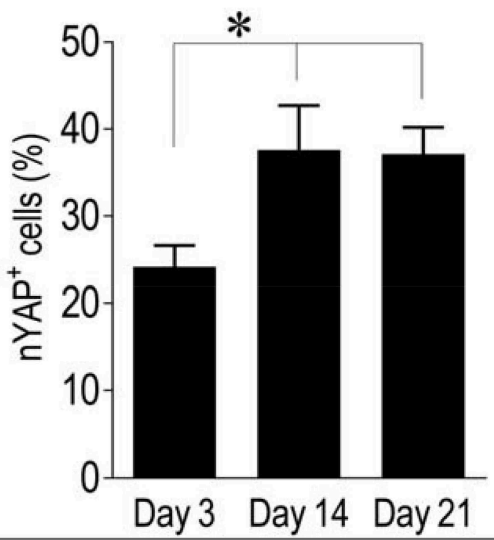

FIGURE 4 | Cellular phenotype and dynamics inside recellularized pericardia. (A-C) Immunofluorescence staining showing expression of $\alpha$ SMA, PCNA, and YAP (all in green fluorescence) in conjunction with Vimentin (in red fluorescence) inside the recellularized pericardial tissue at day 3 and 21 of culturing. In each panel the lower micrographs show the magnification of the boxed insets in the higher photographs. (D) Quantification of total number of cells by DAPI-stained nuclei counting (upper bar graph), $\mathrm{PCNA}^{+}$cells (middle bar graph) and cells with nuclear localized YAP (nYAP ${ }^{+}$cells, low bar graph) at all the considered time points. Statistical analysis was performed with one-way ANOVA with Newman Keuls post-hoc; ${ }^{*}$ indicates $P<0.05(n=3)$.

biogenesis-e.g., Procollagen-Proline-4-Dioxygenase (22), valve maturation-e.g., Biglycan (23), Glycan binding proteins e.g., Galectin (24) or cellular proteins indicative of the valve-interstitial/smooth muscle cells phenotype (e.g., $\alpha$ SMA and $\mathrm{SM} 22 \alpha$ ) at similar levels to the native valves (see also Figure 3B). It was interesting to note that several extracellular 
structural components such as Collagens, Vitronectin, and Fibronectin were higher represented in recellularized samples at day 3 compared to the other conditions (Figure 3C). For a representation of all the differentially expressed proteins with a function in the extracellular matrix present in the native and recellularized pericardial tissue see Figure 3D, where they clustered into two groups, one evidently more abundant at day 3 of recellularization and mostly composed of ECM structural components (Collagens), and another more represented in native valves and day 21 , where matricellular proteins involved in ECM deposition, maturation, remodeling were mostly represented.

In a recent publication of our group (25), we have described that stiffness-dependent pathological activation of human valve interstitial cells is related to the activity of the main transcriptional component of the Hippo pathway, the YAP transcription factor (26). Since YAP nuclear translocation in VICs causes increase in proliferation, we determined the number of cells expressing the Ki-67 cell cycle marker and we correlated this with expression of YAP protein in the cells present in our recellularized pericardium samples. In parallel, we determined the expression of aSMA, one of the pathologic markers associated to VICs transformation into myofibroblasts, and Vimentin, a pan-VIC marker (15). Figure 4 shows the result of immunofluorescence staining of the recellularized pericardium at day 3 and day 21 of culture and quantitation of results of cell counting and determination of nuclear YAP $\left(\mathrm{nYAP}^{+}\right)$and $\mathrm{PCNA}^{+}$ cells. As shown in panel A, double immunofluorescence with the pan-VIC marker Vimentin and aSMA antibodies showed a majority of cells stained with Vimentin and a relatively small number of cells expressing also $\alpha \mathrm{SMA}$, especially confined at the surface of the tissue constructs. The double staining including PCNA and YAP in combination with Vimentin (panels B and C), indicated, respectively, a relatively high level of cells with nuclear staining of PCNA (a characteristic of cells in active cell cycle) and expressing YAP at both time points. These observations were corroborated by determination of $\mathrm{PCNA}^{+}$and $\mathrm{YAP}^{+}$cells percentages showing a time-dependent increase between day 3 and day 21 (panel D). Interestingly, while the total number of cells had a trend to increase (although not significantly) from 14 to 21 days, the percentages of $\mathrm{PCNA}^{+}$and $\mathrm{YAP}^{+}$cells were comparable in pericardial samples at day 14 and day 21 of culture.

\section{DISCUSSION}

The quest for novel applications to replace the conventionally used biological material (aldehyde-treated pericardium) and manufacture cardiac valve replacements has prompted a variety of approaches ranging from "off-the-shelf" solutions based on natural/bioartificial materials, to cell seeding into artificially-designed scaffolds manufactured with mechanical characteristics of the natural valves (27). Importantly, despite these advances, the current biological valve implants are still manufactured with animal-derived pericardium treated with aldehydes, a 50-year dated technology with minimal or null modifications introduced since then (28).

Inspired by evidences showing the feasibility of re-engineering entire organs depleted of their original cellular content by recellularization protocols (29), we have endeavored a tissue engineering program with the aim at generating a valve-compliant tissue that may be employed as a living material for aortic valve reconstruction. This solution might be a viable alternative for patients under the age limits for prosthetic valve implantation (30) or, potentially, for correcting congenital valve defects in alternative to conventional procedures (31). The key features that represent a novelty in our procedure compared to others already employed to resolve this problem (18) are that (i) it does not affect mechanical resistance of the pericardial material $(15,32)$, (ii) it strongly reduces its immunogenic potential by abolishing foreign body reaction (32) and presence of $\alpha \mathrm{GAL}$ xenoantigen (15), and (iii) it increases the permeability of the tissue, making it perfusable to achieve an unprecedented efficiency of recellularization (16).

Despite this work is not the first to employ a bioreactor system to assess cell viability inside a decellularized scaffold for cardiac valve engineering $(33,34)$, it is the first to address the problem of valve maturation of a fully recellularized tissue using a systematic proteomic approach. In fact, our mass spectrometry data clearly showed that compared to the native pericardial membrane, a number of proteins were upregulated in the material as a result of de-novo protein synthesis by the introduced valve cells. This occurred with a reproducible time dependence (Figure 2A) and gave rise to distinct proteomic patterns at the beginning and at the end of the culture period (Figure 2B). It was finally interesting to note that 14 days of culture represents a critical time point in the maturation progress of the tissues (Figure 2A, Figure S1), likely due to transitioning of the cells from an active proliferation to a differentiation phase. This clearly establishes a turning point that might be useful to monitoring the quality of the valve tissue generation process based on assessment of tissue construct protein content at this time point.

By analyzing more in details the proteomic data produced by mass spectrometry, we found three protein clusters that were characterized, respectively by, (i) proteins more abundant in the initial culture stage (day 3) compared with the native valve and the final (day 21) pericardium recellularization stage (the "green" cluster in Figure 2B), (ii) proteins whose representation was higher in the native valves, irrespective of the recellularized pericardium culture time (the "blue" protein cluster in Figure 2B) and (iii) proteins whose abundance was higher in day 21 pericardium culture stage compared to day 3 and, at lower extent, to the native valve (the "red cluster" in Figure 2B).

A higher abundance of matrix structural components in decellularized vs. the native samples at short time after cell seeding (the majority in the green protein cluster, Figures $2 \mathbf{B}$, $\mathbf{3 A}, \mathbf{D})$ is in line with what already reported for the comparison 
between the decellularized vs. the native pericardium samples in our previous investigation, where probably it resulted from increase in the relative content of matrix proteins due to removal of the cellular proteins and GAGs (16) and a too low number of cells (Figure 4D). The same reason may account for the higher presence of other components of the ECM such as Tenascin (Figures 2B, 3C,D) at the same time point. It was finally interesting to note that while Tenascin was present in high amount in the native pericardium and it was removed from decellularization process (16), Thrombospondin was transiently expressed by valve cells at the beginning of the recellularization period. Given the general role of Thrombospondins in pro-fibrotic activation of VICs (35), it is possible that this factor contributes to active migration of these cells inside the decellularized pericardial matrix.

The larger cluster of proteins differentially expressed in the different conditions contained proteins with higher or equal representation in the recellularized pericardium at day 21 of culture compared to the native valves. It was interesting to find in this group (named the "red" cluster), the presence of enzymes involved in ECM maturation and remodeling such as the Procollagen-Proline 2-Oxoglutarate4-Dioxygenase (P4HA1) (22) and the Peptidyl-Prolyl cis-trans-Isomerase (PPIase) (36) or Cathepsin-D (37), of enzymes necessary for cell-ECM interactions such as Protein Disulfide Isomerase (PDI) (38), of proteoglycans involved in valve matrix organization such as Biglycan (23), and, finally of cellular proteins typically expressed in valve interstitial cells such as Transgelin/SM22 $\alpha$ and $\alpha$ SMA (39). Taken together, these results show that the consistent repopulation of the pericardium with valve cells promotes a valve maturation of the tissue constructs, even if, as showed by the absence other specific valve ECM components such as Aggrecan, Osteoglicyn/Mimecan, Decorin (40) at both stages of recellularized pericardium (the "blue" protein cluster in Figures 2A,C, 3D), suggests that the process was still not complete.

It was also interesting to observe that the number of cells in the tissue constructs tended to reach a plateau in the transition from the 14 to the 21 days of culture (Figure 4). This finding is important for the possibility that cells lose control of their proliferation process once they enter in the pericardial matrix, thus causing risks of uncontrollable growth, and disarrangement of the pericardial matrix. In this respect it has to be noted that the number of cells with nucleartranslocated YAP, a transcription factor connected to the propathologic phenotype in human VICs also reached a plateau (Figure 4) and was lower than in portions of human pathologic aortic valves (25), thereby suggesting an equilibrium between control of proliferation vs. acquisition of valve degeneration markers during valve maturation of the tissue constructs. How this is established it is for now only a matter of speculation, even if, in line with our results on human VICs (25), we hypothesize that this may result from mechanical control of YAP nuclear translocation due to relatively low compliance of the decellularized matrix. Further studies will be done to characterize the local stiffness of the pericardial matrix at different times after recellularization.

In summary, we provide the first example of a partial valve tissue maturation process achieved experimentally by seeding valve cells into a decellularized pericardial matrix. We are now planning new studies with human-derived bone marrow/adipose-derived mesenchymal stem cells and scaled up bioreactors able to efficiently repopulate decellularized pericardial patches with surgical size. It will be also interesting to assess whether mounting the recellularized pericardial material onto stent posts and valve pulse duplicators will promote further biological maturation of the living pericardium material due to mechanical conditioning (41), and will maintain the viability of the cells under tissue mechanical stress (33), as a step forward in translation of the living pericardium material for clinical employment.

\section{DATA AVAILABILITY STATEMENT}

The datasets generated for this study can be found in the Zenodo depository: https://doi.org/10.5281/zenodo.3689596.

\section{ETHICS STATEMENT}

Ethical review and approval was not required for the animal study because the porcine material used in the study was obtained at local slaughterhouses.

\section{AUTHOR CONTRIBUTIONS}

FA, MBa, GB, and NS performed experiments. SR helped in paper revision. $\mathrm{MBr}$ and $\mathrm{CB}$ analyzed data. GP designed the experiments. MP designed the experiments, analyzed data, and wrote the paper.

\section{FUNDING}

The present work has been funded by Institutional project grants at Centro Cardiologico Monzino (Ricerca Corrente; Progetto 5 per 1000), issued to MP.

\section{SUPPLEMENTARY MATERIAL}

The Supplementary Material for this article can be found online at: https://www.frontiersin.org/articles/10.3389/fcvm. 2020.00080/full\#supplementary-material

Figure S1 | Clusterization analysis of proteomic data including samples from all the time points. As shown in the PC analysis (Figure 2A), the protein content in day 14 samples showed a higher variability consistent with day 14 being a critical time point for maturation of the cellularized pericardia samples.

Table S1 | List of the differentially expressed proteins revealed by MS. Besides the accession number of each of them it is indicated the result of variance calculation, the fold change, the protein description, and the highest mean condition, colored with the same code adopted for protein clusters represented in Figure 2B. 


\section{REFERENCES}

1. David TE. Surgical treatment of aortic valve disease. Nat Rev Cardiol. (2013) 10:375-86. doi: 10.1038/nrcardio.2013.72

2. Kheradvar A, Groves E, Goergen C, Alavi SH, Tranquillo R, Simmons C, et al. Emerging trends in heart valve engineering: part II. novel and standard technologies for aortic valve replacement. Ann Biomed Eng. (2014) 43:844-57. doi: 10.1007/s10439-014-1191-5

3. Forcillo J, Pellerin M, Perrault LP, Cartier R, Bouchard D, Demers $\mathrm{P}$, et al. Carpentier-edwards pericardial valve in the aortic position: 25-Years experience. Ann Thorac Surg. (2013) 96:486-93. doi: 10.1016/j.athoracsur.2013.03.032

4. Grabenwoger M, Sider J, Fitzal F, Zelenka C, Windberger U, Grimm M, et al. Impact of glutaraldehyde on calcification of pericardial bioprosthetic heart valve material. Ann Thorac Surg. (1996) 62:772-7.

5. Siddiqui RF, Abraham JR, Butany J. Bioprosthetic heart valves: modes of failure. Histopathology. (2009) 55:135-44. doi: 10.1111/j.1365-2559.2008.03190.x

6. Galili U. The [alpha]-gal epitope and the anti-Gal antibody in xenotransplantation and in cancer immunotherapy. Immunol Cell Biol. (2005) 83:674-86. doi: 10.1111/j.1440-1711.2005.01366.x

7. Konakci ZK, Bohle B, Blumer R, Hoetzenecker W, Roth G, Moser B, et al. Alpha-Gal on bioprostheses: xenograft immune response in cardiac surgery. Eur J Clin Invest. (2005) 35:17-23. doi: 10.1111/j.1365-2362.2005.01441.x

8. Hülsmann J, Grün $\mathrm{K}$, El Amouri S, Barth $\mathrm{M}$, Hornung $\mathrm{K}$, Holzfuß C, et al. Transplantation material bovine pericardium: biomechanical and immunogenic characteristics after decellularization vs glutaraldehyde-fixing. Xenotransplantation. (2012) 19:286-97. doi: 10.1111/j.1399-3089.2012.00719.x

9. Naso F, Gandaglia A, Iop L, Spina M, Gerosa G. Alpha-Gal detectors in xenotransplantation research: a word of caution. Xenotransplantation. (2012) 19:215-20. doi: 10.1111/j.1399-3089.2012.00714.x

10. Naso F, Gandaglia A, Bottio T, Tarzia V, Nottle MB, D'apice AJF, et al. First quantification of alpha-Gal epitope in current glutaraldehyde-fixed heart valve bioprostheses. Xenotransplantation. (2013) 20:252-61. doi: 10.1111/xen. 12044

11. Jana S, Tefft BJ, Spoon DB, Simari RD. Scaffolds for tissue engineering of cardiac valves. Acta Biomater. (2014) 10:2877-93. doi: $10.1016 /$ j.actbio.2014.03.014

12. Kheradvar A, Groves E, Dasi L, Alavi SH, Tranquillo R, Grande-Allen KJ, et al. Emerging trends in heart valve engineering: part I. Solutions for future. Ann Biomed Eng. (2014) 43:833-43. doi: 10.1007/s10439-014-1209-z

13. Van Loosdregt IAEW, Argento G, Driessen-Mol A, Oomens CWJ, Baaijens FPT. Cell-mediated retraction versus hemodynamic loading - a delicate balance in tissue-engineered heart valves. J Biomech. (2014) 47:2064-9. doi: 10.1016/j.jbiomech.2013.10.049

14. Van Vlimmeren MA, Driessen-Mol A, Oomens CW, Baaijens FP. An in vitro model system to quantify stress generation, compaction, and retraction in engineered heart valve tissue. Tissue Eng Part C Methods. (2011) 17:983-91. doi: 10.1089/ten.tec. 2011.0070

15. Santoro R, Consolo F, Spiccia M, Piola M, Kassem S, Prandi F, et al. Feasibility of pig and human-derived aortic valve interstitial cells seeding on fixative-free decellularized animal pericardium. J Biomed Mat Res Part B-App Biomater. (2016) 104:345-56. doi: 10.1002/jbm.b.33404

16. Amadeo F, Boschetti F, Polvani G, Banfi C, Pesce M, Santoro R. Aortic valve cell seeding into decellularized animal pericardium by perfusion-assisted bioreactor. J Tissue Eng Regen Med. (2018) 12:1481-93. doi: 10.1002/ter m. 2680

17. Dainese L, Guarino A, Burba I, Esposito G, Pompilio G, Polvani $\mathrm{G}$, et al. Heart valve engineering: decellularized aortic homograft seeded with human cardiac stromal cells. J Heart Valve Dis. (2012) 21: 125-34.

18. Vedepo MC, Detamore MS, Hopkins RA, Converse GL. Recellularization of decellularized heart valves: progress toward the tissue-engineered heart valve. J Tissue Eng. (2017) 8:2041731417726327. doi: 10.1177/20417314177 26327

19. Brioschi M, Lento S, Tremoli E, Banfi C. Proteomic analysis of endothelial cell secretome: a means of studying the pleiotropic effects of Hmg-CoA reductase inhibitors. J Proteomics. (2013) 78:346-61. doi: 10.1016/j.jprot.2012. 10.003

20. Brioschi M, Eligini S, Crisci M, Fiorelli S, Tremoli E, Colli S, et al. A mass spectrometry-based workflow for the proteomic analysis of in vitro cultured cell subsets isolated by means of laser capture microdissection. Anal Bioanal Chem. (2014) 406:2817-25. doi: 10.1007/s00216-014-7724-9

21. Roverso M, Brioschi M, Banfi C, Visentin S, Burlina S, Seraglia R, et al. A preliminary study on human placental tissue impaired by gestational diabetes: a comparison of gel-based versus gel-free proteomics approaches. Eur J Mass Spectrom. (2016) 22:71-82. doi: 10.1255/ejms.1412

22. Gilkes DM, Bajpai S, Chaturvedi P, Wirtz D, Semenza GL. Hypoxia-inducible factor 1 (HIF-1) promotes extracellular matrix remodeling under hypoxic conditions by inducing P4HA1, P4HA2, and PLOD2 expression in fibroblasts. J Biol Chem. (2013) 288:10819-29. doi: 10.1074/jbc.M112.442939

23. Stephens EH, Post AD, Laucirica DR, Grande-Allen KJ. Perinatal changes in mitral and aortic valve structure and composition. Pediatr Dev Pathol. (2010) 13:447-58. doi: 10.2350/09-11-0749-OA.1

24. Yang R-Y, Rabinovich GA, Liu F-T. Galectins: structure, function and therapeutic potential. Expert Rev Mol Med. (2008) 10:e17. doi: $10.1017 /$ S1462399408000719

25. Santoro R, Scaini D, Severino LU, Amadeo F, Ferrari S, Bernava G, et al. Activation of human aortic valve interstitial cells by local stiffness involves YAP-dependent transcriptional signaling. Biomaterials. (2018) 181:268-79. doi: 10.1016/j.biomaterials.2018.07.033

26. Dupont S, Morsut L, Aragona M, Enzo E, Giulitti S, Cordenonsi M, et al. Role of YAP/TAZ in mechanotransduction. Nature. (2011) 474:179-83. doi: 10.1038/nature 10137

27. Jover E, Fagnano M, Angelini G, Madeddu P. Cell sources for tissue engineering strategies to treat calcific valve disease. Front Cardiovasc Med. (2018) 5:155. doi: 10.3389/fcvm.2018.00155

28. Li KYC. Bioprosthetic heart valves: upgrading a 50-year old technology. Front Cardiovasc Med. (2019) 6:47. doi: 10.3389/fcvm.2019.00047

29. Ott HC, Matthiesen TS, Goh SK, Black LD, Kren SM, Netoff TI, et al. Perfusion-decellularized matrix: using nature's platform to engineer a bioartificial heart. Nat Med. (2008) 14:213-21. doi: 10.1038/nm1684

30. Head SJ, Celik M, Kappetein AP. Mechanical versus bioprosthetic aortic valve replacement. Eur Heart J. (2017) 38:2183-91. doi: 10.1093/eurheartj/ehx141

31. Sologashvili T, Pretre R. The place of the ozaki procedure in the treatment of aortic valve disease. Swiss Med Wkly. (2018) 148:w14612. doi: 10.4414/smw.2018.14612

32. Vinci MC, Tessitore G, Castiglioni L, Prandi F, Soncini M, Santoro R, et al. Mechanical compliance and immunological compatibility of fixativefree decellularized/cryopreserved human pericardium. PLOS ONE. (2013) 8:e64769. doi: 10.1371/journal.pone.0064769

33. Kennamer A, Sierad L, Pascal R, Rierson N, Albers C, Harpa M, et al. Bioreactor conditioning of valve scaffolds seeded internally with adult stem cells. Tissue Eng Regen Med. (2016) 13:507-15. doi: 10.1007/s13770-016-9114-1

34. Gonzalez BA, Issa EP, Mankame OV, Bustillos J, Cuellar A, Rodriguez AJ, et al. Porcine small intestinal submucosa mitral valve material responses support acute somatic growth. Tissue Eng A. (2020). doi: 10.1089/ten.tea.2019.0220. [Epub ahead of print].

35. Pohjolainen V, Mustonen E, Taskinen P, Näpänkangas J, Leskinen $\mathrm{H}$, Ohukainen $\mathrm{P}$, et al. Increased thrombospondin-2 in human fibrosclerotic and stenotic aortic valves. Atherosclerosis. (2012) 220:66-71. doi: 10.1016/j.atherosclerosis.2011.10.003

36. Lin W, Bonin M, Boden A, Wieduwild R, Murawala P, Wermke M, et al. Peptidyl prolyl cis/trans isomerase activity on the cell surface correlates with extracellular matrix development. Commun Biol. (2019) 2:58. doi: 10.1038/s42003-019-0315-8

37. Langley SR, Willeit K, Didangelos A, Matic LP, Skroblin P, BarallobreBarreiro J, et al. Extracellular matrix proteomics identifies molecular signature of symptomatic carotid plaques. J Clin Invest. (2017) 127:1546-60. doi: 10.1172/JCI86924

38. Rosenberg N, Mor-Cohen R, Sheptovitsky VH, Romanenco O, Hess O, Lahav J. Integrin-mediated cell adhesion requires extracellular disulfide exchange regulated by protein disulfide isomerase. Exp Cell Res. (2019) 381:77-85. doi: 10.1016/j.yexcr.2019.04.017 
39. Latif N, Quillon A, Sarathchandra P, Mccormack A, Lozanoski A, Yacoub $\mathrm{MH}$, et al. Modulation of human valve interstitial cell phenotype and function using a fibroblast growth factor 2 formulation. PLoS ONE. (2015) 10:e127844. doi: 10.1371/journal.pone.01 27844

40. Roosens A, Handoyo YP, Dubruel P, Declercq H. Impact of modified gelatin on valvular microtissues. J Tissue Eng Regen Med. (2019) 13:771-84. doi: 10.1002/term.2825

41. Cox MAJ, Kortsmit J, Driessen N, Bouten CVC, Baaijens FPT. Tissueengineered heart valves develop native-like collagen fiber architecture. Tissue Eng Part A. (2009) 16:1527-37. doi: 10.1089/ten.tea.2009.0263
Conflict of Interest: The authors declare that the research was conducted in the absence of any commercial or financial relationships that could be construed as a potential conflict of interest.

Copyright (C) 2020 Amadeo, Barbuto, Bernava, Savini, Brioschi, Rizzi, Banfi, Polvani and Pesce. This is an open-access article distributed under the terms of the Creative Commons Attribution License (CC BY). The use, distribution or reproduction in other forums is permitted, provided the original author(s) and the copyright owner(s) are credited and that the original publication in this journal is cited, in accordance with accepted academic practice. No use, distribution or reproduction is permitted which does not comply with these terms. 